\title{
Outcomes of Neonates Born to Mothers With Coronavirus Disease 2019 (COVID-19) - National Neonatology Forum (NNF) India COVID-19 Registry
}

\author{
National NeOnatology Forum (NNF) COVID-19 Registry Group* \\ *List of group members provided as Annexure \\ Correspondence to: Professor Praveen Kumar, Head, Division of Neonatology, Department of Pediatrics, Postgraduate Institute of \\ Medical Education and Research (PGIMER), Chandigarh,India.drpkumarpgi@gmail.com \\ Received: January 27, 2021; Initial review: February 25, 2021; Accepted: March 13, 2021.
}

Background: Limited evidence exists on perinatal transmission and outcomes of severe acute respiratory syndrome coronavirus2 (SARS-CoV-2) infection in neonates.

Objective: To describe clinical outcomes and risk factors for transmission in neonates born to mothers with perinatal SARSCoV-2 infection.

Design: Prospective cohort of suspected and confirmed SARSCoV-2 infected neonates entered in National Neonatology Forum (NNF) of India registry.

Subjects: Neonates born to women with SARS-CoV-2 infection within two weeks before or two days after birth and neonates with SARS-CoV-2 infection.

Outcomes: Incidence and risk factors of perinatal transmission.

Results: Among 1713 neonates, SARS-CoV-2 infection status was available for 1330 intramural and 104 extramural neonates. SARS-CoV-2 positivity was reported in 144 intramural and 39 extramural neonates. Perinatal transmission occurred in 106 $(8 \%)$ and horizontal transmission in $21(1.5 \%)$ intramural neonates. Neonates roomed-in with mother had higher transmission risk (RR1.16, 95\% Cl 1.1 to $2.4 ; P=0.01$ ). No association was noted with the mode of delivery or type of feeding. The majority of neonates positive for SARS-CoV2 were asymptomatic. Intramural SARS-CoV-2 positive neonates were more likely to be symptomatic (RR $5,95 \% \mathrm{Cl} 3.3$ to $7.7 ; P<0.0001)$ and need resuscitation (RR 2, $95 \% \mathrm{Cl} 1.0$ to $3.9 ; P=0.05$ ) compared to SARS-CoV-2 negative neonates. Amongst symptomatic neonates, most morbidities were related to prematurity and perinatal events.

Conclusion: Data from a large cohort suggests perinatal transmission of SARS-CoV-2 infection and increased morbidity in infected infants.

Keywords: Horizontal transmission, Outcome, Perinatal transmission, Risk.

Published online: March 20, 2021;PII: S097475591600300
$\mathrm{T}$ he severe acute respiratory syndrome coronavirus-2 (SARS-CoV-2) has infected over 15 million individuals in India [1]. The SARS-CoV-2 infects both children and adults but has higher fatality in the elderly and individuals with co-morbidities [2]. SARS-CoV-2 infects pregnant women as much as other reproductive-age women [3]. The knowledge about the epidemiology, clinical characteri-stics, prevention, and treatment of SARS-CoV-2 infection is continually evolving. Currently available data on the consequences of SARS-CoV-2 infection in pregnancy, fetus, and the neonate is mostly from case reports, small case series, retrospective cohort or cross-sectional studies, compiled in a recent systematic review [4]. There is limited data on perinatal SARS-CoV-2 infection from the developing world. We report analysis from a large neonatal coronavirus disease 2019 (COVID-19) registry under the National Neonatology Forum (NNF) of India, on the incidence of perinatal transmission and the factors associated with it, and the clinical features of SARS$\mathrm{CoV}-2$ positive neonates.

\section{METHODS}

In this prospective cohort study, data were collected from various hospitals voluntarily enrolled in the NNF COVID-19 registry, which was initiated in April, 2020. Neonates born to women with SARS-CoV-2 infection within two weeks prior to or two days after delivery and neonates with confirmed SARS-CoV-2 infection within 28 days of life were eligible for enrolment in the study. COVID status of mothers and neonates was assessed by nasopharyngeal RT-PCR in all participating hospitals.

SARS-CoV-2 infected neonates were defined as those with a positive SARS-CoV-2 quantitative RT-PCR test in nasopharyngeal swab within 28 days of birth [5]. SARSCoV-2 infected mothers were defined as those with a positive SARS-CoV-2 quantitative RT-PCR test in the 
nasopharyngeal sample during the peripartum period [5]. Perinatal transmission was defined as positive nasopharyngeal RT-PCR in a neonate in the first 72 hours after birth [6,7]. This included intrauterine and intrapartum transmission. Testing was avoided in the first 12 hours to minimize false positives due to superficial colonization. Horizontal transmission was considered in a neonate with negative RT-PCR within the first 72 hours who subsequently tested positive any time after 72 hours of birth irrespective of the mother's SARS-CoV-2 status [6,7].

Mothers were either tested at admission or referred for admission because of the SARS-CoV-2 positive reports. Testing and management were as per the Indian Council for Medical Research (ICMR) and NNF guidelines, and local standard operating procedures [8,9]. SARS-CoV-2 status of the neonate was tested as per NNF guidelines and local institutional protocols, which in most centres was done within the first 72 hours after birth or when the neonate was symptomatic and admitted to the neonatal unit [8]. All neonates were monitored for clinical symptoms for the first seven days after birth and for as long as the mother was admitted to the hospital. Data on mothers and neonates was available till disposition from the hospital.

Data collection: Data was prospectively submitted to the registry by the participating hospitals in a web-based secure platform (https://innc.org/covid). All the registered hospitals received a short message alert every week on the number of enrolled cases and data completeness. Twenty percent of the enrolled cases from each participating centre were randomly cross-checked for data quality. A closed group of clinical leads from participating hospitals was formalized for secure, encrypted communication. This group managed the updates to the database, day-to-day problems in data management, the progress of the registry, and sharing of education and communication. Any inconsistency in data was highlighted to the respective hospital lead for verification.

The data included baseline characteristics of the mothers, mode of delivery, type of feeding, rooming-in with mother or isolation from mother, clinical features, diagnosis, and neonatal outcomes. The clinical status of patients was classified as per guidelines from the Ministry of Health and Family Welfare, Government of India [10].

The primary outcomes of the study were: $(i)$ the incidence of perinatal transmission; (ii) the rates of SARS-CoV-2 virus positivity in the neonates in association with risk factors of transmission such as mode of delivery, type of feeding and care practices, and (iii) comparisons between intramural and extramural, SARS-
CoV-2 positive and SARS-CoV-2 negative neonates born to SARS-CoV-2 positive mothers. The secondary outcomes were respiratory morbidities, the need for respiratory support, and mortality in these infants.

Statistical analyses: Descriptive statistics were used, and comparisons were made using the chi-square test for categorical variables and the Student t-test or MannWhitney $U$ test as appropriate for continuous variables. A $P$-value of $<0.05$ was considered significant.

\section{RESULTS}

The web-based COVID-19 registry received a total of 1733 entries for mothers and their neonates. Almost all (1730/1733) of the enrolled mother-infant dyads were from tertiary care hospitals, 1649 (95\%) from public sector hospitals, and 82 (5\%) from private hospitals. We excluded 22 entries where both mothers and neonates were negative but were referred because of suspicion of SARS-CoV-2 infection, and 1711 mother-infant dyads were enrolled in this study. Most of the mothers (94.5\%) were asymptomatic, and only $1 \%$ were critically ill. Cesarean section was the mode of delivery in $68 \%$.

Of the 1711 enrolled neonates, 1589 were intramural, while 122 were extramural births. The extramural infants were referred to the participating hospitals for either symptomatic status or for SARS-CoV-2 positive status of the mother. Figure 1 gives a study flow for intramural neonates enrolled in the registry. Out of 1589 intramural infants, SARS-CoV-2 testing was either not done or not reported in 259, so we excluded them from the analysis. Amongst 1330 tested neonates, 143 (10.8\%) were SARSCoV-2 positive. Of these, $68(5.1 \%)$ tested positive on day one (Fig. 1 and 2). Amongst the intramural newborn infants, $106(8 \%)$ were positive for SARS-CoV-2 within 72 hours (perinatal transmission) and $21(1.5 \%)$ beyond 72 hours (horizontal transmission) of birth. The risk of transmission was not associated with the mode of delivery or type of feeding. The risk of transmission of SARS-CoV2 from mother to neonate was marginally higher if the baby was roomed-in with the mother (RR 1.16, 95\% CI 1.1-2.4; $P=0.01)$. Tables I and II compare the SARS-CoV-2 positive and negative infants born to SARS-CoV-2 positive mothers. The demographic para-meters in both the groups were not different except for the prematurity rate, which was higher in SARS-CoV-2 positive group. SARS-CoV-2 positive neonates were five times more likely to be symptomatic and twice more likely to need resuscitation. They had significantly higher probability of having sepsis and septic shock. SARS-CoV-2 positive neonates were more likely to have abnormal radiological findings and need respiratory support. They were also more likely to have received surfactant, steroids and inotropes. The risk of 


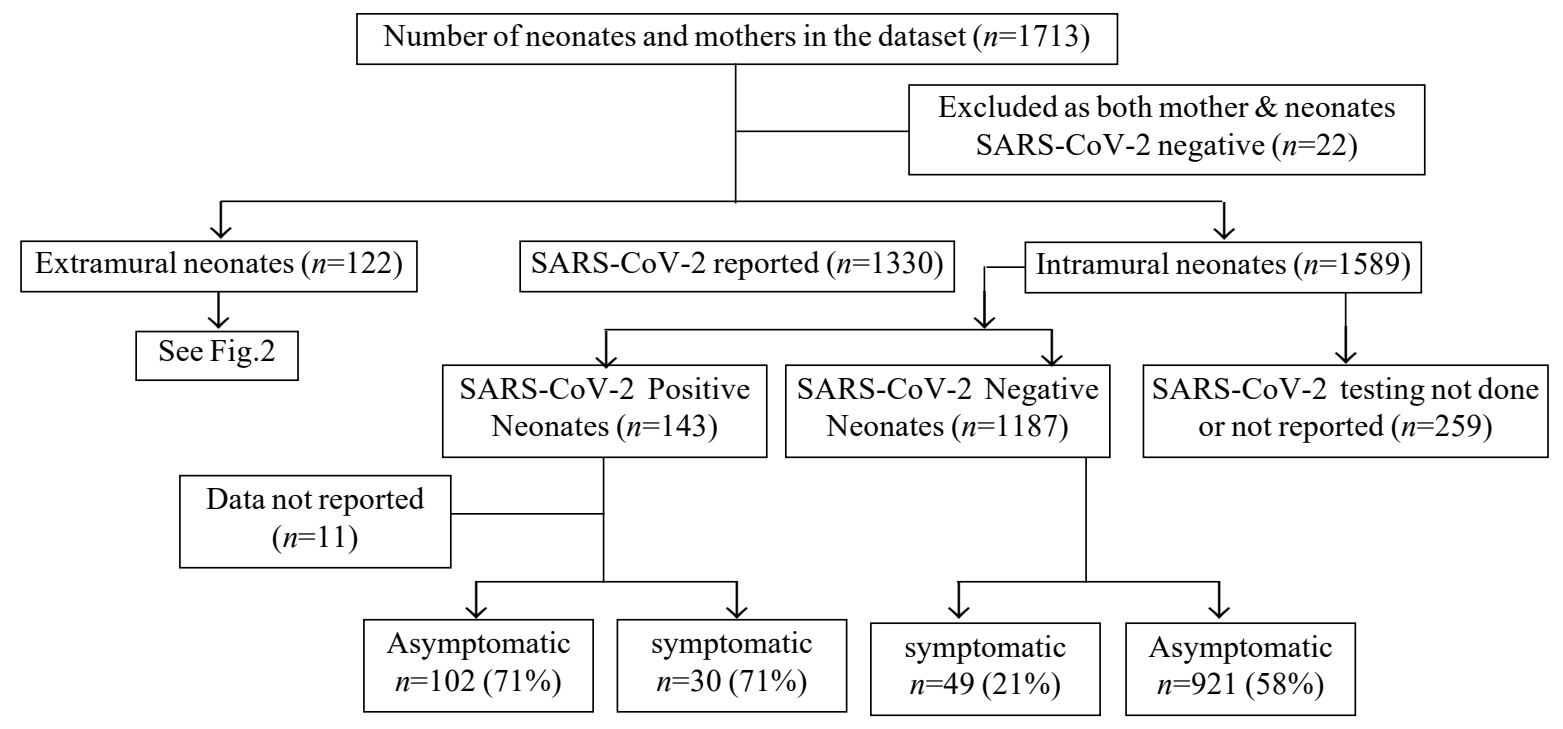

Number of SARS-CoV-2 positive neonates according to the day of testing: Day 1,68; Day 2,8; Day 3,30.

Perinatal transmission $(<72$ h) - 106/1330 (8\%); Number of neonates positive for SARS-CoV-2 beyond $72 \mathrm{~h}$ of life - 21/1330 (1.5\%)

Fig. 1 Study population flow for intramural neonates.

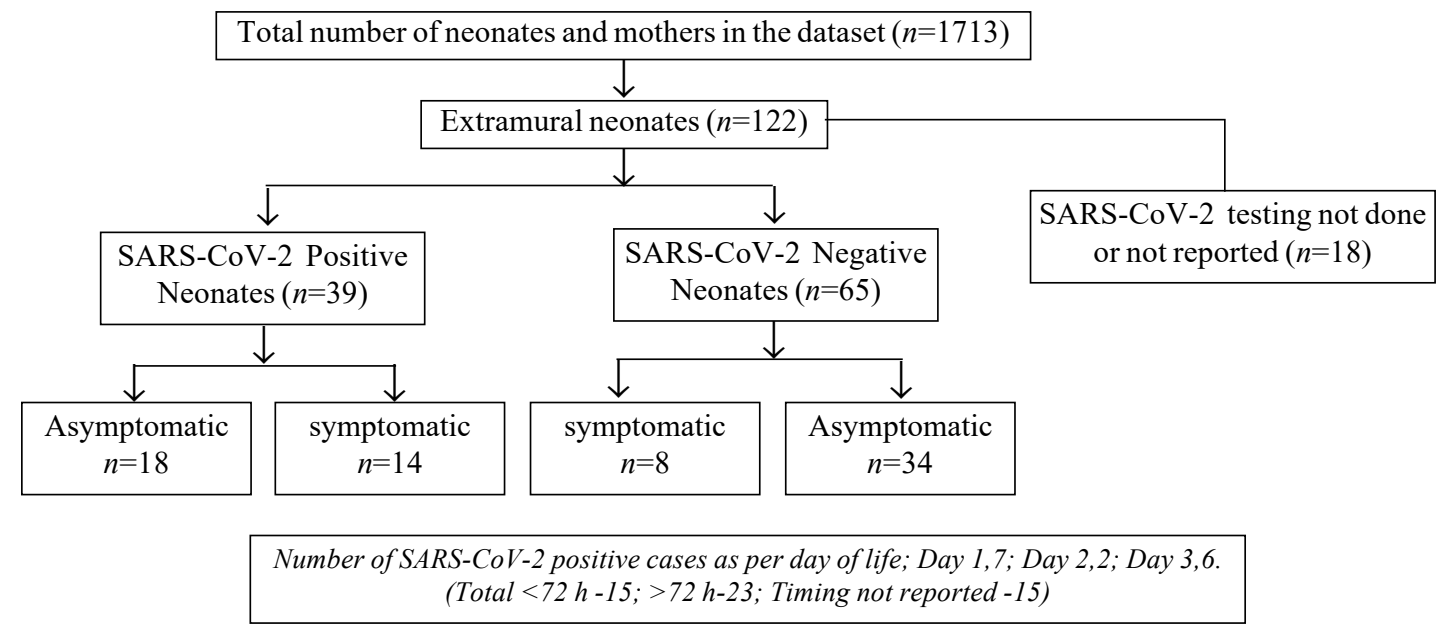

Fig. 2 Study population flow for extramural neonates.

mortality was however not significantly different between the two groups. No significant association was noted between mother's symptomatic status and baby's SARSCoV-2 positivity, need for resuscitation, and symptoms.

Fig. 2 depicts the study flow for extramural neonates. Extramural SARS-CoV-2 infected neonates were more likely to present with pneumonia, seizures, and septic shock, and were more likely to present after the first 72 hours of birth (Tables III and IV). This cohort of neonates was generally symptomatic. Like intramural neonates, extramural SARS-CoV-2 infected neonates tended to have more respiratory symptoms, radiological abnormalities, and needed more respiratory support.

There were 17 neonates in the registry, who were positive for SARS-CoV-2, but their mothers were reported negative. Five of these neonates were positive within 72 hours of life, and the remaining tested positive beyond 72 hours of birth. In this sub-group, 3 (17.6\%) needed resuscitation at birth, $3(17.6 \%)$ had pneumonia, 5 (29\%) had respiratory distress, and $4(23.5 \%)$ had sepsis. One (5.9\%) infant had encephalopathy, and 3 (17.6\%) had seizures. 
Table I Demographics and Risk Factors in SARS-CoV-2 Positive and Negative Intramural Neonates

\begin{tabular}{|c|c|c|c|}
\hline \multirow[t]{2}{*}{ Parameters } & \multicolumn{2}{|c|}{$S A R S-C o V-2$} & \multirow[t]{2}{*}{$R R(95 \% C I)$} \\
\hline & $\begin{array}{l}\text { Positive } \\
(n=143)\end{array}$ & $\begin{array}{l}\text { Negative } \\
(n=1187)\end{array}$ & \\
\hline Male gender & $81(57)$ & $581(49.2)$ & $1.1(0.9-1.3)$ \\
\hline Weight, $\mathrm{g}^{a}$ & $2746(618)$ & $3024(170)$ & \\
\hline Gestation, $\mathrm{wk}^{a}$ & $37.5(2.1)$ & $37.7(1.5)$ & \\
\hline Prematurity & $\begin{array}{l}\text { Reported in } \\
130(90.9)\end{array}$ & $\begin{array}{l}\text { Reported in } \\
1142(96.2)\end{array}$ & \\
\hline $34-36$ wk & $21(16.1)$ & $106(8.9)$ & $1.6(1-2.5)^{c}$ \\
\hline$<34$ wk & $6(4.6)$ & $15(1.3)$ & $3.3(1.3-8.4)^{b}$ \\
\hline$<37$ wk & $27(20.7)$ & $121(10.2)$ & $1.8(1.2-2.7)^{b}$ \\
\hline Caesarean delivery & $88(61.5)$ & $821(69.7)$ & $0.8(0.7-1)$ \\
\hline Mother positive $^{d}$ & $135(94.4)$ & $1167(98.3)$ & $0.1(0.08-0.12)^{b}$ \\
\hline $\begin{array}{l}\text { Roomed-in with } \\
\text { mother }\end{array}$ & $111(77.6)$ & $800(67.4)$ & $1.16(1.1-2.4)^{c}$ \\
\hline Breastfeeding & $119(83.2)$ & $998(84.1)$ & $0.97(0.6-1.5)$ \\
\hline
\end{tabular}

Values in no. (\%) or amean (SD); ${ }^{b} P<0.01 ;{ }^{c} P=0.01$; ${ }^{d} R T-P C R$ for SARS-CoV-2.

\section{DISCUSSION}

In this large registry of neonates born to SARS-CoV-2 positive mothers, we report the incidence of neonatal infection, type of symptoms, and neonatal outcomes. Neonates acquired infection most commonly in the first $72 \mathrm{~h}$ after birth. The $5.1 \%$ neonates who tested positive on day one may have acquired the infection intrauterine or intrapartum. Those neonates who tested positive on days two or three may also have been due to intrauterine transmission but could have acquired the infection postnatally from mother, other family members, or healthcare providers. In the absence of serial testing and testing of various body fluids from mother, it is not possible to pinpoint the timing of acquisition. It is also to be noted that many neonates were tested for the first time on day three as per the local protocols, and the absence of testing on the first day after birth could have led to misclassification of the type of infection. We found a significantly higher incidence of perinatal transmission than that reported in a recent review [10].

This study highlights that SARS-CoV-2 positive neonates are more likely to be symptomatic, more likely to have respiratory symptoms, and other neonatal morbidities. However, the mortality is not increased significantly. In a systematic review by Raschetti, et al. [4], the median age at diagnosis was five days, and 55\% of neonates were symptomatic [4]. Common symptoms reported include respiratory distress, fever, and those related to gastrointestinal illness. Most of the infected
Table II Clinical Features and Management of SARS-CoV-2 Infection in Intramural Neonates at Birth

\begin{tabular}{|c|c|c|c|}
\hline \multirow[t]{2}{*}{ Parameters } & \multicolumn{2}{|c|}{$S A R S-C o V-2$} & \multirow[t]{2}{*}{$R R(95 \% C I)$} \\
\hline & $\begin{array}{l}\text { Positive } \\
(n=143)\end{array}$ & $\begin{array}{l}\text { Negative } \\
(n=1187)\end{array}$ & \\
\hline Resuscitation $^{d}$ & $15(10.4)$ & $26(2.1)$ & $4.4(2.4-8.2)^{a}$ \\
\hline Symptomatic & $30(21)$ & $49(4.1)$ & $5(3.3-7.7)^{a}$ \\
\hline RDS & $13(9)$ & $12(1.1)$ & $7.5(3.4-16.8)^{a}$ \\
\hline Pneumonia & $10(7)$ & $1(0.08)$ & $83(10.7-643)^{a}$ \\
\hline Sepsis & $5(3.5)$ & $1(0.08)$ & $41.5(4.8-352)^{a}$ \\
\hline Seizures & $5(3.5)$ & $6(0.5)$ & $6.9(2.1-22.3)^{b}$ \\
\hline Septic Shock & $6(4.2)$ & $6(0.5)$ & $8.3(2.7-25.3)^{a}$ \\
\hline DIC & $4(2.8)$ & $4(0.3)$ & $8.3(2-32)^{c}$ \\
\hline Encephalopathy & $3(2.1)$ & $7(0.6)$ & $3.5(0.93-13.6)$ \\
\hline Jaundice & $6(4.2)$ & $6(0.5)$ & $8.3(2.7-25.4)^{a}$ \\
\hline Other morbidities & $28(19)$ & $43(4.5)$ & $5.4(3.4-8.4)^{a}$ \\
\hline Abnormal CXR & $16(11.2)$ & $7(0.6)$ & $18.9(7.9-45.3)^{a}$ \\
\hline Oxygen therapy & $11(7.7)$ & $27(2.3)$ & $3.3(1.7-6.6)^{a}$ \\
\hline CPAP & $7(4.9)$ & $13(1)$ & $4.5(1.8-11)^{a}$ \\
\hline Ventilation & $8(5.6)$ & $14(1.2)$ & $4.7(2-11.1)^{a}$ \\
\hline Surfactant & $5(3.5)$ & $7(0.6)$ & $5.9(1.9-18.4)^{c}$ \\
\hline Inotropes & $8(5.6)$ & $10(0.8)$ & $6.6(2.6-16.5)^{a}$ \\
\hline Corticosteroids & $4(2.8)$ & $2(0.2)$ & $16.6(3-89.8)^{b}$ \\
\hline IVIG & 0 & $1(0.08)$ & - \\
\hline Oseltamivir & $2(1.4)$ & 0 & . \\
\hline Mortality & $2(1.4)$ & $4(0.3)$ & $4.1(0.76-22.4)$ \\
\hline
\end{tabular}

${ }^{a} P<0.001 ;{ }^{b} P=0.001 ;{ }^{c} P<0.05 ;{ }^{d}$ at birth. CXR: chest X-ray; IVIG: intravenous immunoglobulin, CPAP: continuous positive airway pressure; RDS: respiratory distress syndrome; DIC: disseminated intravascular coagulation.

Table III Demographics and Risk Factors in SARS-CoV-2 Positive and Negative Extramural Neonates

\begin{tabular}{|c|c|c|}
\hline \multirow[t]{2}{*}{ Parameters } & \multicolumn{2}{|c|}{$S A R S-C o V-2$} \\
\hline & $\begin{array}{l}\text { Positive } \\
(n=39)\end{array}$ & $\begin{array}{r}\text { Negative } \\
\quad(n=65)\end{array}$ \\
\hline Male gender & $22(57)$ & $32(49.2)$ \\
\hline Weight $(\mathrm{g})^{a}$ & $2572(600)$ & $2822(582)$ \\
\hline Gestation (wk) ${ }^{a}$ & $36.9(2.2)$ & $37.3(1.7)$ \\
\hline Prematurity & 27 & 35 \\
\hline 34-36 weeks & $4(14.8)$ & $2(5.7)$ \\
\hline$<34$ weeks & $3(4.6)$ & $4(1.3)$ \\
\hline Total $<37$ wk & $7(19.4)$ & $6(7 \%)$ \\
\hline Caesarean delivery & $17(43.6)$ & $43(66.1)$ \\
\hline Mother positive $\mathrm{e}^{b, d}$ & $26(76.5)$ & $62(95.4)$ \\
\hline Roomed-in & $17(43.6)$ & $23(35.9)$ \\
\hline Breastfeeding ${ }^{c}$ & $18(48.6)$ & $45(70.3)$ \\
\hline
\end{tabular}


Table IV Clinical Features and Management of SARS-CoV-2 Infection in Extramural Neonates

\begin{tabular}{|c|c|c|c|}
\hline \multirow[t]{2}{*}{ Parameters } & \multicolumn{2}{|c|}{$S A R S-C o V-2$} & \multirow[t]{2}{*}{$R R(95 \% C I)$} \\
\hline & $\begin{array}{c}\text { Positive } \\
(n=39)\end{array}$ & $\begin{array}{l}\text { Negative } \\
(n=65)\end{array}$ & \\
\hline Symptomatic & $13 / 27(48)$ & $6 / 36(16.6)$ & $2.9(1.2-6.6)^{d}$ \\
\hline RDS & $13(33.3)$ & $8(12.2)$ & $2.7(1.2-5.9)^{c}$ \\
\hline Pneumonia & $6(15.4)$ & $1(1.5)$ & $10.1(1.2-81)^{c}$ \\
\hline Seizures & $4(10.2)$ & $1(1.5)$ & $6.6(0.7-57)^{d}$ \\
\hline Septic Shock & $6(15.4)$ & $1(1.5)$ & $10(1.2-80)^{d}$ \\
\hline DIC & $3(7.7)$ & 0 & $12.1(0.6-229)^{e}$ \\
\hline Encephalopathy & $1(2.6)$ & $1(1.5)$ & $1.6(0.1-25.8)^{e}$ \\
\hline Diarrhoea & $2(5.1)$ & $2(3.1)$ & $1.6(0.2-11.3)^{e}$ \\
\hline Other morbidities & $11(28)$ & $2(3.1)$ & $9.1(2.1-39)^{b}$ \\
\hline Abnormal CXR & $16(11.2)$ & $7(0.6)$ & $18.9(7.9-45.3)^{a}$ \\
\hline Oxygen therapy & $10(25.6)$ & $4(6.1)$ & $4.1(1.4-12.3)^{c}$ \\
\hline CPAP & $3(7.7)$ & $3(4.6)$ & $1.7(0.3-8.2)^{e}$ \\
\hline Ventilation & $7(17.9)$ & $1(1.5)$ & $11.6(1.5-91)^{c}$ \\
\hline Inotropes & $5(12.8)$ & $1(1.5)$ & $7.7(0.9-64)^{e}$ \\
\hline Corticosteroids & $2(5.1)$ & 0 & $8.2(0.4-167)^{e}$ \\
\hline IVIG & $2(5.1)$ & 0 & $8.2(0.4-167)^{e}$ \\
\hline Mortality & $1(2.6)$ & $1(1.5)$ & $1.6(0.1-25.8)^{e}$ \\
\hline
\end{tabular}

neonates were not reported to need any respiratory support and had a good outcome after a median duration of hospitalization of 10 days. In our study cohort, $21 \%$ (30/143) of SARS-CoV-2 positive intramural neonates were symptomatic, and the most common symptoms included respiratory distress and sepsis-like features. However, fever and gastrointestinal symptoms were not commonly reported. The prematurity rate of $20.7 \%$ in our cohort was significant and similar to what was reported by the UK registry [11]. This raises concerns about the possibility of increased risk of premature labor in SARSCoV-2 positive pregnant women. The incidence of symptomatic infection reported by us is lower than that reported in the previous reviews [4,12-13] and an Indian case series [14], but similar to that reported by Anand, et al. [15]. This can be explained by possible selection bias inherent in the type of studies - case reports and case series- included in the systematic reviews.

Another important finding in our cohort is that SARSCoV-2 infected neonates were significantly more likely to need resuscitation, be symptomatic, need NICU admi- ssion, have abnormal chest $X$-rays, and need respiratory support. Previous studies have reported a high incidence of NICU admissions in SARS-CoV-2 positive neonates or neonates born to SARS-CoV-2 infected mothers [4]. Some of the variations in NICU admission rates may be due to local protocols for admission and isolation rather than due to the illness per se. Prematurity is an obvious confounder for higher respiratory and other morbidities in the SARS-CoV-2 positive group, and we did not adjust for prematurity rates. However, the difference in prematurity rates is unlikely to explain the magnitude of differences in morbidities.

We found a marginally higher incidence of infection in neonates who were roomed-in with mother. However, we did not find any association with breastfeeding. Similar findings have been reported by Raschetti, et al. [4], wherein lack of mother-neonate separation from birth was associated with late SARS-CoV-2 infections, while breastfeeding was not associated with increased risk. Recent systematic reviews have found a very low rate of detection of SARS-CoV-2 RNA in breastmilk, with a much higher prevalence of antibodies to the virus in the breastmilk $[16,17]$. The World Health Organization recommends that neonates should be roomed-in with mother and exclusively breastfed while following precautions to limit the spread of SARS-CoV-2 infection to neonates [18]. A higher incidence of neonatal infection in infants roomed-in with mothers is likely due to incomplete adherence to the suggested precautions. Salvatore, et al. found no perinatal transmission in a cohort of 116 SARS-CoV-2 positive pregnant women from three New York hospitals with rooming-in and breastfeeding, if correct hygine precautions, maternal masking and parental education were undertaken [19]. Similarly, Anand, et al. [15] report a low risk of transmission of infection from mother to baby with rooming-in and breastfeeding. This is important information for families to be aware of, along with the finding that infection is asymptomatic in the majority of neonates, and the outcome is largely favorable. Meanwhile, more research is needed to evaluate measures to prevent postnatal transmission to neonates and improve adherence to currently prescribed precautions.

We also report on extramural neonates referred to tertiary care hospitals following contact with an infected adult in the family or for other morbidities. SARS-CoV-2 positivity was more likely if these neonates were symptomatic at admission. The repertoire of symptoms was similar to symptomatic intramural neonates. The high incidence of infection in neonates who are symptomatic at presentation underscores the need for universal testing guidelines for this category of neonates [20]. 


\section{WHAT IS ALREADY KNOWN?}

- Limited evidence exists on the perinatal transmission and the management of severe acute respiratory syndrome coronavirus 2 (SARS-CoV-2) infection among newborns, especially from the developing world.

\section{WHAT THIS STUDY ADDS?}

- Our data confirms perinatal transmission of SARS-CoV-2 and suggests increased morbidity in infected infants. Breastfeeding and rooming-in seem to be safe but require compliance with additional precautions.

This large registry was created for the new COVID19 disease within a short span of time after the WHO's announcement of the pandemic and data was contributed by 20 hospitals on a voluntary basis across the country. The most important limitation, as highlighted above, was non-uniformity in age at testing of neonates born to SARS-CoV-2 positive mothers. As this was a registrybased study, testing of other biological sources like amniotic fluid, placenta, blood, or breastmilk was not pursued. We also did not test for the presence of specific antibodies in the neonatal blood to look for intrauterine infection as suggested by a recent guideline to classify the type of neonatal infection [7]. We did not capture data for neonates with the possibility of multi-system inflammatory syndrome in children (MIS-C) following SARSCoV-2 infection, which is recently being reported [21].

In conclusion, our study provides important data on neonatal infection, clinical features, and outcomes in neonates born to SARS-CoV-2 positive women. This information can be used to make informed decisions and policies on neonatal SARS-CoV-2 testing, healthcare organization for neonates born to SARS-CoV-2 positive women, and counseling of families regarding various management options.

Acknowledgements: Bodhgire Sachin and Aradhana Mishra, Government Medical College, Aurangabad, Maharashtra; Tanmay Sar and Bilkish Islam, Nil Ratan Sircar Medical College, Kolkata, West Bengal; Vivek Kumar and Dhirendra Prasad Yadav, Department of Pediatrics, All India Institute of Medical Sciences, New Delhi; Md Zakiulla and Purbasha Mishra, All India Institute of Medical Sciences, Bhubaneswar, Odisha.

Contributors: KM: initiated and wrote the proposal for the registry and created a data collection form, analyzed the data, and finalized the first draft of the manuscript; BT, SM and PK: facilitated the creation of the data registry on web-portal hosted by the Indian Neonatal Collaborative (INCC), India; BT: coordinated data collection and helped to extract relevant data from the portal; SM: wrote the introduction and methods sections, helped in biostatistics and approved the final draft of the manuscript; DC: reviewed the analysis, results section and wrote an interpretation of data and discussion; AD: approved the proposal, encouraged and coordinated hospitals' participation through National Neonatology Forum, India and reviewed the final draft of the manuscript; PK: approved the proposal, created a registry on the portal, encouraged hospitals' participation and approved the final draft of the manuscript. All the remaining Collaborators contributed significantly towards data collection and sharing from their respective institutes, and also reviewed and approved the final draft of the manuscript.

Funding: None; Competing interest: None stated.

\section{REFERENCES}

1. COVID19 India. Accessed 20 April, 2021. Available at https://www.mohfw.gov.in/

2. Guan WJ, Ni ZY, Hu Y, et al. Clinical characteristics of coronavirus disease 2019 in China. N Engl J Med. 2020; 382:1708-720.

3. Fox NS, Melka S. COVID-19 in pregnant women: Case series from one large New York city obstetrical practice. Am J Perinatol. 2020;37:1002-04.

4. Raschetti R, Vivanti AJ, Vauloup-Fellous C, et al. Synthesis and systematic review of reported neonatal SARS-CoV-2 infections. Nature Communications. 2020;11: 5164.

5. Sheth S, Shah N, Bhandari V. Outcomes in COVID-19 positive neonates and possibility of viral vertical transmission: A narrative review. Am J Perinatol. 2020;37: 1208-16.

6. Blumberg DA, Underwood MA, Hedriana HL, et al. Vertical Transmission of SARS-CoV-2: What is the Optimal Definition? Am J Perinatol. 2020;37:769-72.

7. Shah PS, Diambomba Y, Acharya G, et al. Classification system and case definition for SARS-CoV-2 infection in pregnant women, fetuses, and neonates. Acta Obstet Gynecol Scand. 2020;99:565-68.

8. Chawla D, Chirla D, Dalwai S, et al. Perinatal-Neonatal Management of COVID-19 Infection - Guidelines of the Federation of Obstetric and Gynaecological Societies of India (FOGSI), National Neonatology Forum of India (NNF), and Indian Academy of Pediatrics (IAP). Indian Pediatr. 2020;57:536-48.

9. Ministry of Health and Family Welfare. Guidelines for Management of COVID-19. Accessed January 05, 2021. Available at https://www.mohfw.gov.in

10. Dhir SK, Kumar J,Meena J, Kumar P. Clinical features and outcome of SARS-CoV-2 infection in neonates: A systematic review. J Trop Pediatr. 2020 Aug 28: fmaa059.

11. Gale C, Quigley MA, Placzek A, et al. Characteristics and outcomes of neonatal SARS-cov-2 infection in the UK: A prospective national cohort study using active surveillance. Lancet Child Adolesc Health. 2021;5:113-121. 
12. Kotlyar AM, Grechukhina O, Chen A, et al. Vertical transmission of coronavirus disease 2019: A systematic review and meta-analysis. Am J Obstet Gynecol. 2021; 224: 35-53.

13. Walker KF, O'Donoghue K, Grace N, et al. Maternal transmission of SARS-COV-2 to the neonate, and possible routes for such transmission: A systematic review and critical analysis. BJOG. 2020;127:1324-36.

14. Nanavati R, Mascarenhas D, Goyal M, et al. A single-center observational study on clinical features and outcomes of 21 SARS-cov-2-infected neonates from India. Eur J Pediatr. 2021;1-12.

15. Anand P, Yadav A, Debata $P$, et al. Clinical profile, viral load, management and outcome of neonates born to COVID 19 positive mothers: A tertiary care centre experience from India. Eur J Pediatr. 2021;180:547-59.

16. Kumar J, Meena J, Yadav A, et al. SARS-CoV-2 detection in human milk: A systematic review. J Matern Fetal Neonatal Med. 2021 Feb 8;1-8.

17. Zhu F, Zozaya C, Zhou Q, et al. SARS-CoV-2 genome and

ANNEXURE

\section{Members of the National Neonatology Forum (NNF) COVID-19 Registry Group}

\section{Writing Group}

Kiran More, Sidra Medicine Hospital, Doha, Qatar; Deepak Chawla, Government Medical College, Chandigarh; Srinivas Murki, Paramitha Children Hospital, Hyderabad, Telangana; Baswaraj Tandur, Princess Durru Shehvar Children's and General Hospital; Ashok K Deorari, All India Institute of Medical Sciences, New Delhi; Praveen Kumar, Postgraduate Institute of Medical Education and Research, Chandigarh.

\section{Investigators}

Suchitra Dontamala, Gandhi Hospital and Medical College, Hyderabad, Telangana; Mangalabharathi Sundaram, Institute of Obstetrics and Gynecology, Madras Medical College, Chennai, Tamilnadu; M Anitha, Chengalpattu Medical College and Hospital, Chengalpattu, Tamilnadu; Deshmukh Laxmikant, Government Medical College, Aurangabad, Maharashtra; Asim Kumar Mallick, Nilratan Sircar Medical College and Hospital, Kolkata, West Bengal; Jagjit Singh Dalal, Pt. B.D. Sharma Postgraduate Institute of Medical Sciences, Rohtak, Haryana; Ankit Verma, Department of Pediatrics, All India Institute of Medical Sciences, New Delhi; Vinay Kumar R, SNR Hospital, Kolar, Karnataka; Shilpa Kalane, Deenanath Mangeshkar Hospital, Pune, Maharashtra; Ratan K Das, IMS and SUM antibodies in breastmilk : A systematic review and metaanalysis. Arch Dis Child Fetal Neonatal Ed. 2021 Feb 9; 0F1-F8.

18. World Health Organization. Breastfeeding and COVID-19. Scientific Brief. 23 June 2020. Accessed December 25, 2020. Available at: https://www.who.int/opublications/i/ item/WHO-2019-nCoV-Sci_Brief-Breastfeeding-2020.1

19. Salvatore CM, Han JY, Acker KP, et al. Neonatal management and outcomes during the COVID-19 pan-demic: an observation cohort study. Lancet Child Adolesc Health. 2020;4:721-27.

20. Sivanandan S, Chawla D, Kumar P, Deorari AK, National Neonatology Forum India. COVID-19 in neonates: A call for standardized testing. Indian Pediatr. 2020;57: 1166-71.

21. Nakra NA, Blumberg DA, Herrera-Guerra A, et al. MultiSystem Inflammatory syndrome in children (MIS-C) following SARS-CoV-2 infection: Review of clinical presentation, hypothetical pathogenesis, and proposed management. Children (Basel). 2020;7:69.
Hospital, Bhubaneswar, Odisha; Tapas Kumar Som, All India Institute of Medical Sciences, Bhubaneswar, Odisha; Somosri Ray, Medical College and Hospital, Kolkata, West Bengal; Manish Mittal, Cocoon Hospital, Jaipur, Rajasthan; Ashish Mehta, Arpan Newborn care Centre, Ahmedabad, Gujarat; Chandra Kumar Natarajan, Kanchi Kamakoti Childs Trust Hospital, Chennai, Tamilnadu; Abhishek Aradhya, Ovum Woman and Child Specialty Hospital, Hoskote, Karnataka; Niraj Patel, Shree Navajivan Children Hospital, Rajkot, Gujarat.

\section{Co-Investigators}

JN George and Srikanth Sandanala, Gandhi Medical College and hospital, Hyderabad, Telangana; Mohammed Sajjid and Vijaya Subramanian, Institute of Obstetrics and Gynecology, Madras Medical College, Chennai; Manikumar S, Chengalpattu Medical College and Hospitals, Chengalpattu, Tamil Nadu; Debasis Maity, Nil Ratan Sircar Medical College, Kolkata, West Bengal; Sandeep Jhajra Dayanand, Pt. BD Sharma Postgraduate Institute of Medical Sciences, Rohtak; Balasundar, Nodal Officer, SNR Hospital, Kolar, Karnataka; Rajan V Joshi, Department of Paediatrics, Deenanath Mangeshkar Hospital, Pune, Maharashtra; Pankaj Kumar Mohanty and Tanushree Sahoo, All India Institute of Medical Sciences, Bhubaneswar, Odisha; Dinesh Munian, Medical College and Hospital, Kolkata, West Bengal; Binoy Shah, Arpan Newborn care Centre, Ahmedabad, Gujarat; Vaanathi Vijayakumar, Kanchi Kamakoti Childs Trust Hospital, Chennai, Tamil Nadu. 